\title{
Dielectric sea-ice properties examined by GNSS reflectometry: Findings of the MOSAiC expedition
}

M. Semmling ${ }^{1}$, J. Wickert ${ }^{2,3}$, F. Kreß ${ }^{2,3}$, M. M. Hoque ${ }^{1}$, D. V. Divine ${ }^{4}$, S. Gerland ${ }^{4}$

1 Institute for Solar-Terrestrial Physics, Neustrelitz (DLR-SO)

${ }^{2}$ German Research Centre for Geosciences, Potsdam (GFZ) 3 Technische Universität Berlin (TUB)

4 Norwegian Polar Institute, Tromsø (NPI)

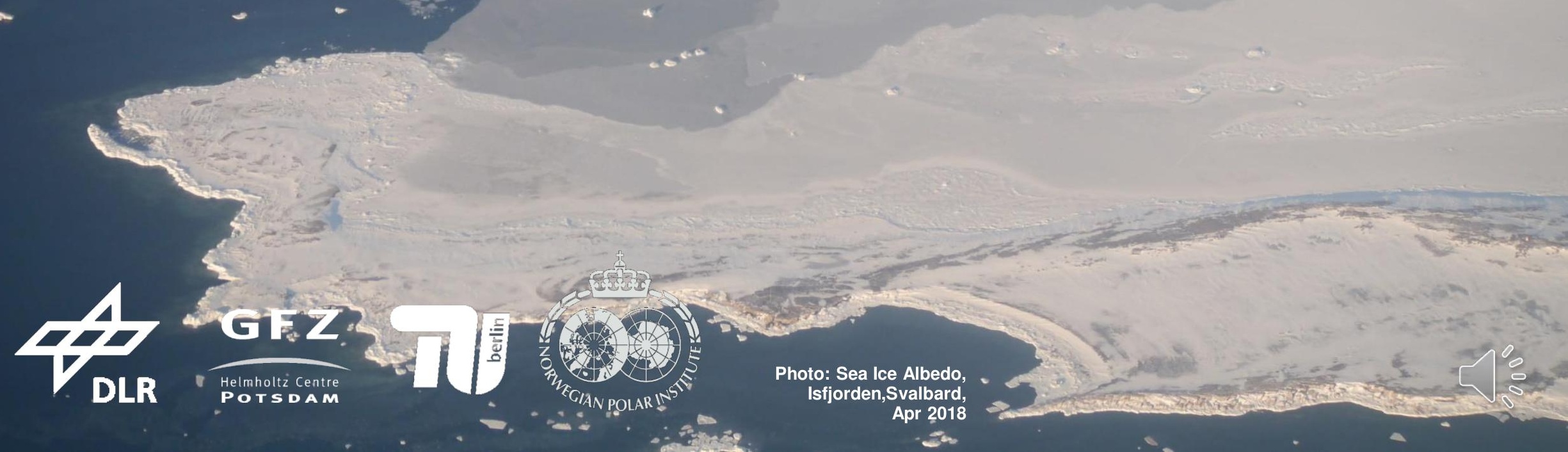




\section{Outline}

1. Introduction

Previous Fram Strait Study

Motivation for Sea-Ice Reflectometry

2. Measurement \& Model

Polarstern Measurements

Reflection Models

3. Results for MOSAiC (first leg)

Reflectivity Profiles

Inverted Permittivity

Slope Anomalies

4. Summary \& Conclusions 


\section{Introduction}

$$
\frac{\mathbf{G F} Z}{\substack{\text { Helmholtz centre } \\ \text { Po T S D A M }}}
$$




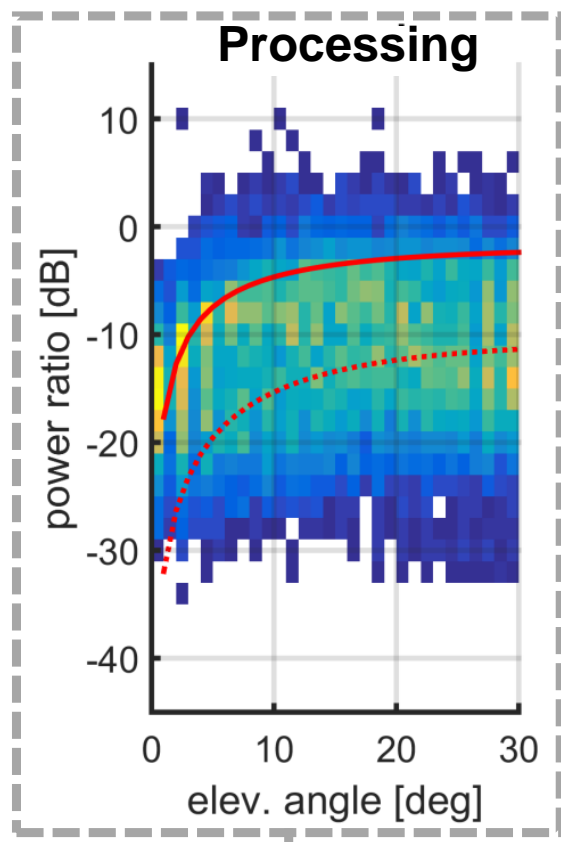

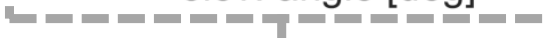

\section{Previous Fram Strait Study}

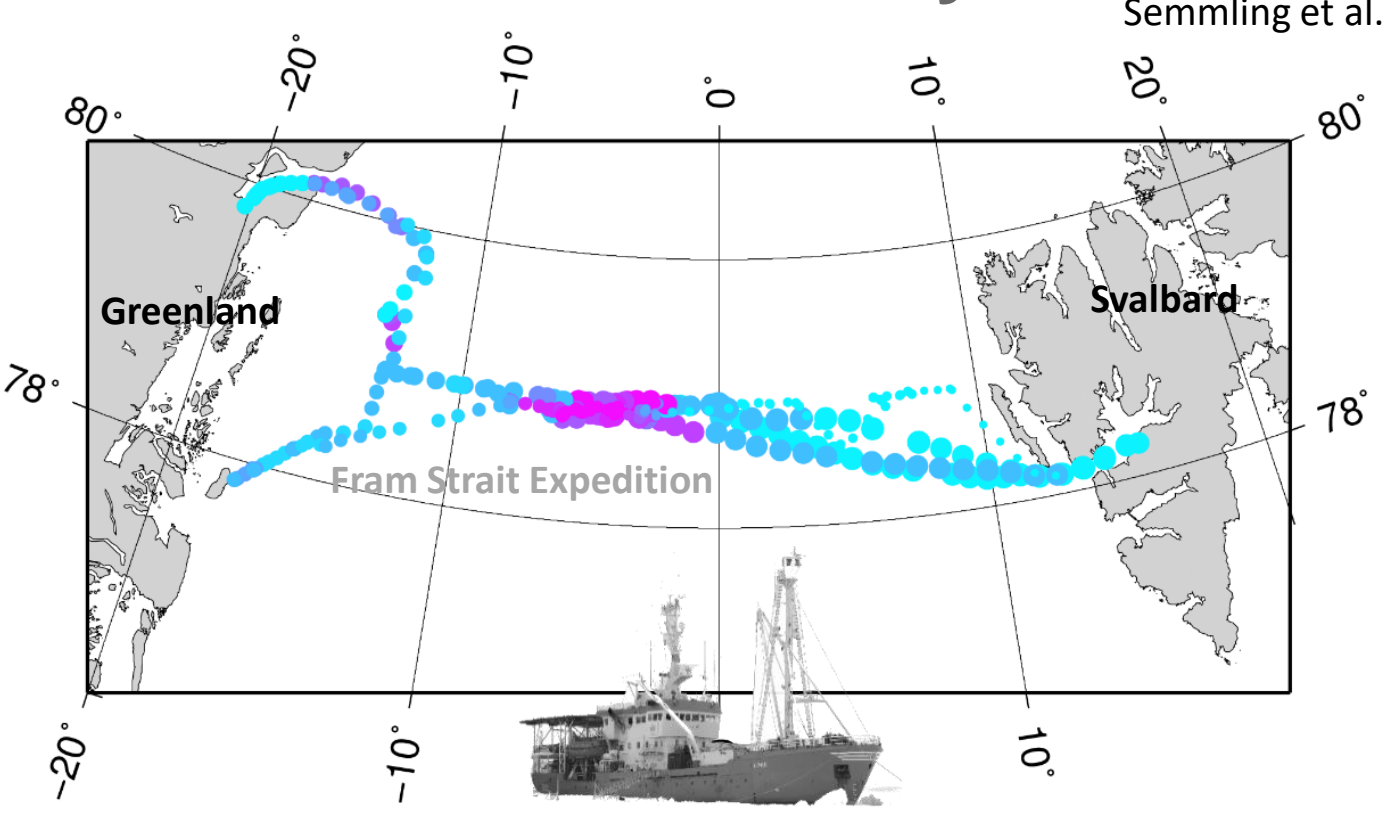

sea ice concentration
$[\%]$

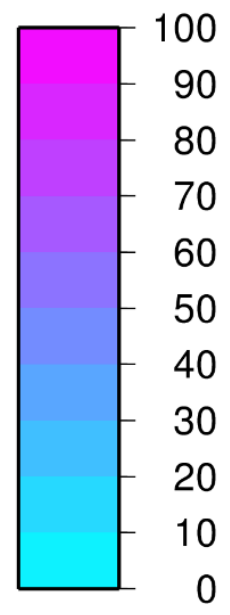

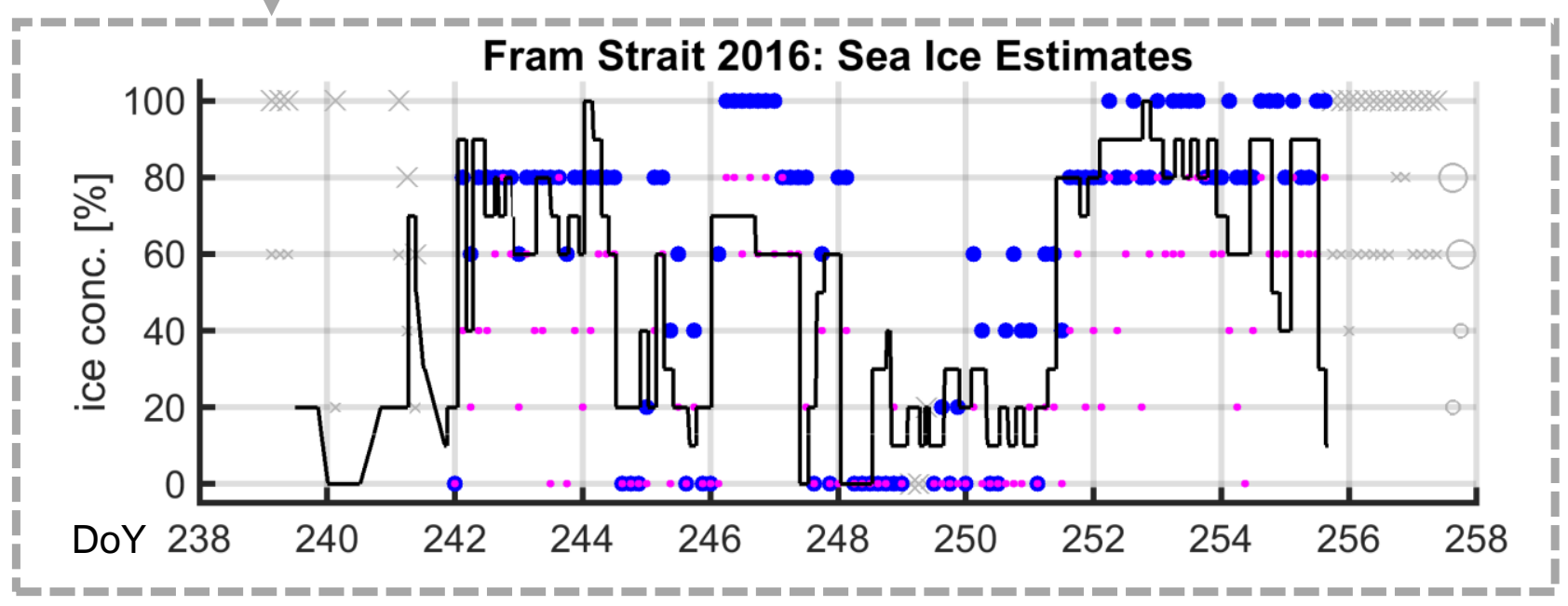

Next opportunity MOSAiC

International Arctic Drift

Expedition

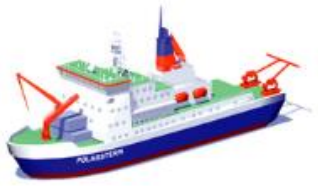

QA $\mathrm{M} / \mathrm{GRAPHIC}$ 


\section{Motivation for Sea-Ice Reflectometry}

1. Importance of Sea Ice:

- crucial effect on Earth's radiation budget

- crucial factor for maritime activities in the Arctic

2. Challenge for GNSS reflectometry:

- sea-ice concentration from permittivity contrast

- retrieve permittivity level from sea-ice type?

- relation of reflectivity and sea-ice thickness?

3. Opportunity of MOSAiC expedition:

- R/V Polarstern as platform for one year Arctic operations

- access to ice deep in central Arctic 


\section{Measurement \& Model}

\section{GF Z




\section{Polarstern Measurements}

MOSAiC expedition 1st leg:

- from Troms $\varnothing$ into the central Arctic

- 86 days (Sep 20 - Dec 14, 2019)

- data permission after Sep 26

Marginal Ice Zone (MIZ):

- Siberian sec. lat. $82^{\circ} \mathrm{N}$ to $85^{\circ} \mathrm{N}$

- 3 days (Sep $28-30,2019$ )

- variable sea-ice concentration

\section{Central Arctic (CA):}

- during/after mooring to ice floe

- 14 days (Dec 1 - 14, 2019)

- permanently compact sea ice

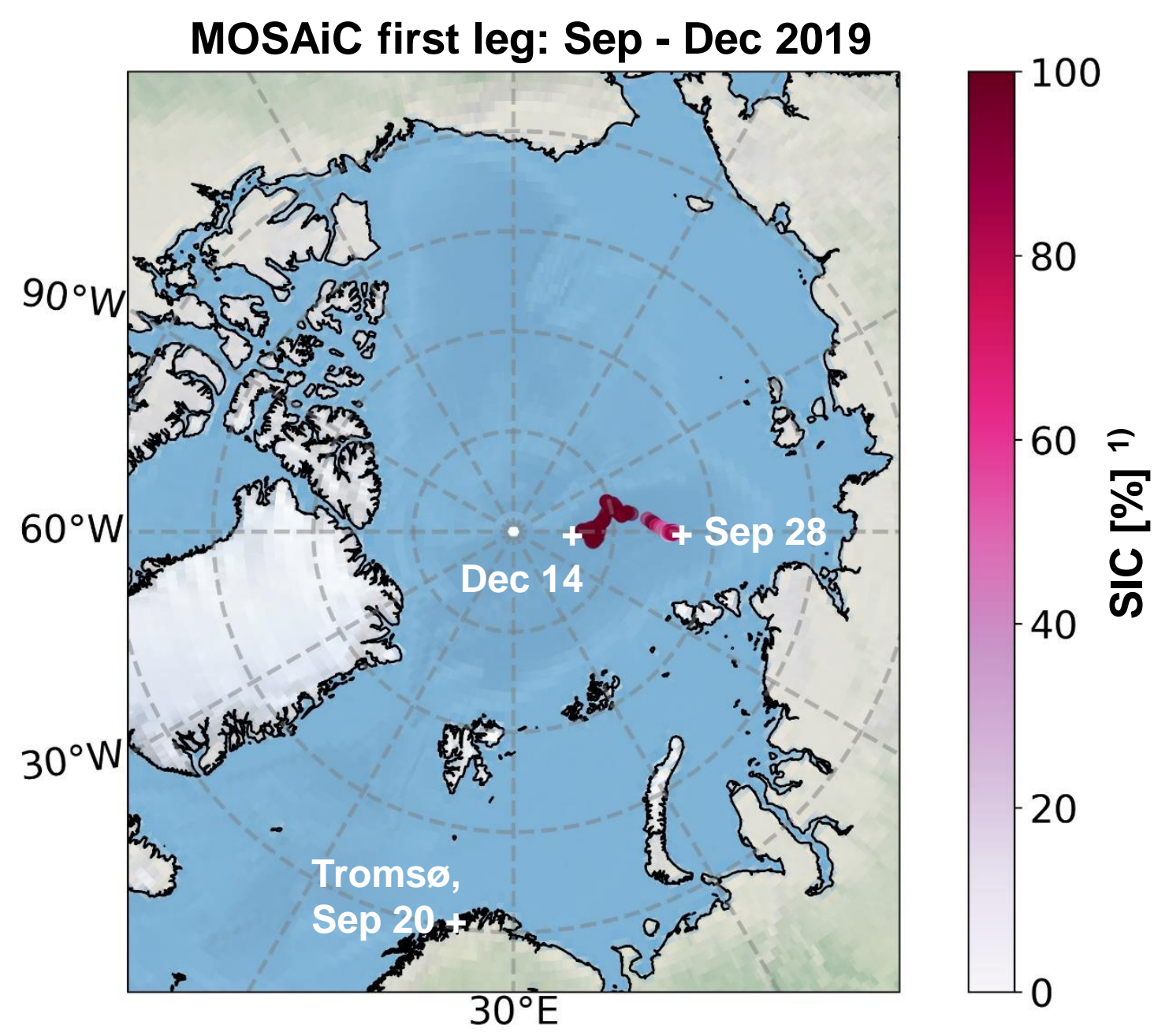

1) ASSIST 2016

\section{G F Z

DLR Helmholtz Centre 


\section{Polarstern Measurements}

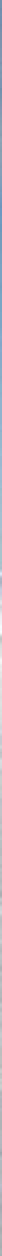




\section{Reflection Model}

Semmling et al. 2019

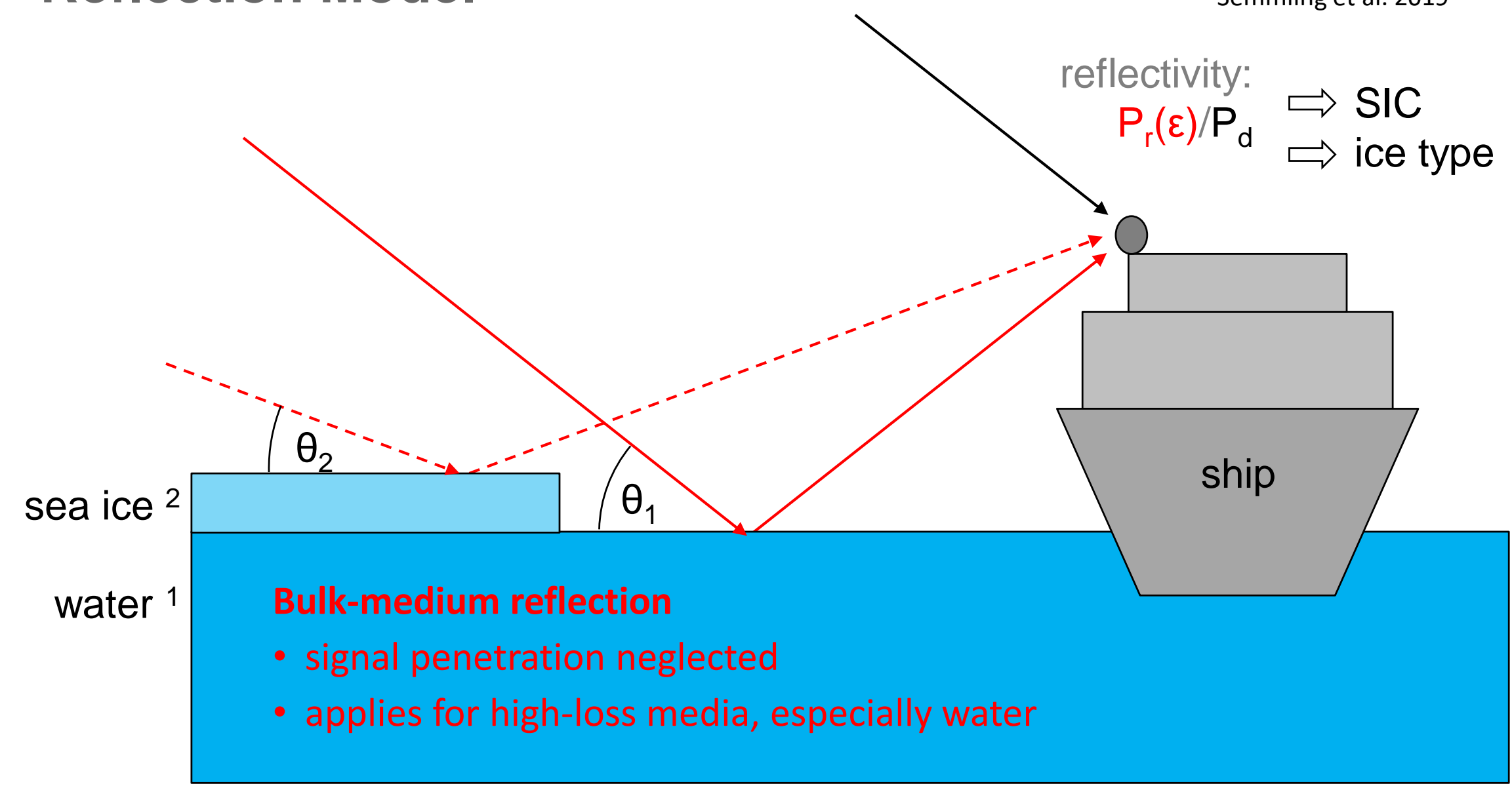

rel. permittivity: $\varepsilon_{1}=76.4+\mathrm{i} 48.5 ; \quad \varepsilon_{2}=3.31+\mathrm{i} 0.11$

\section{GF Z




\section{Reflection Model}

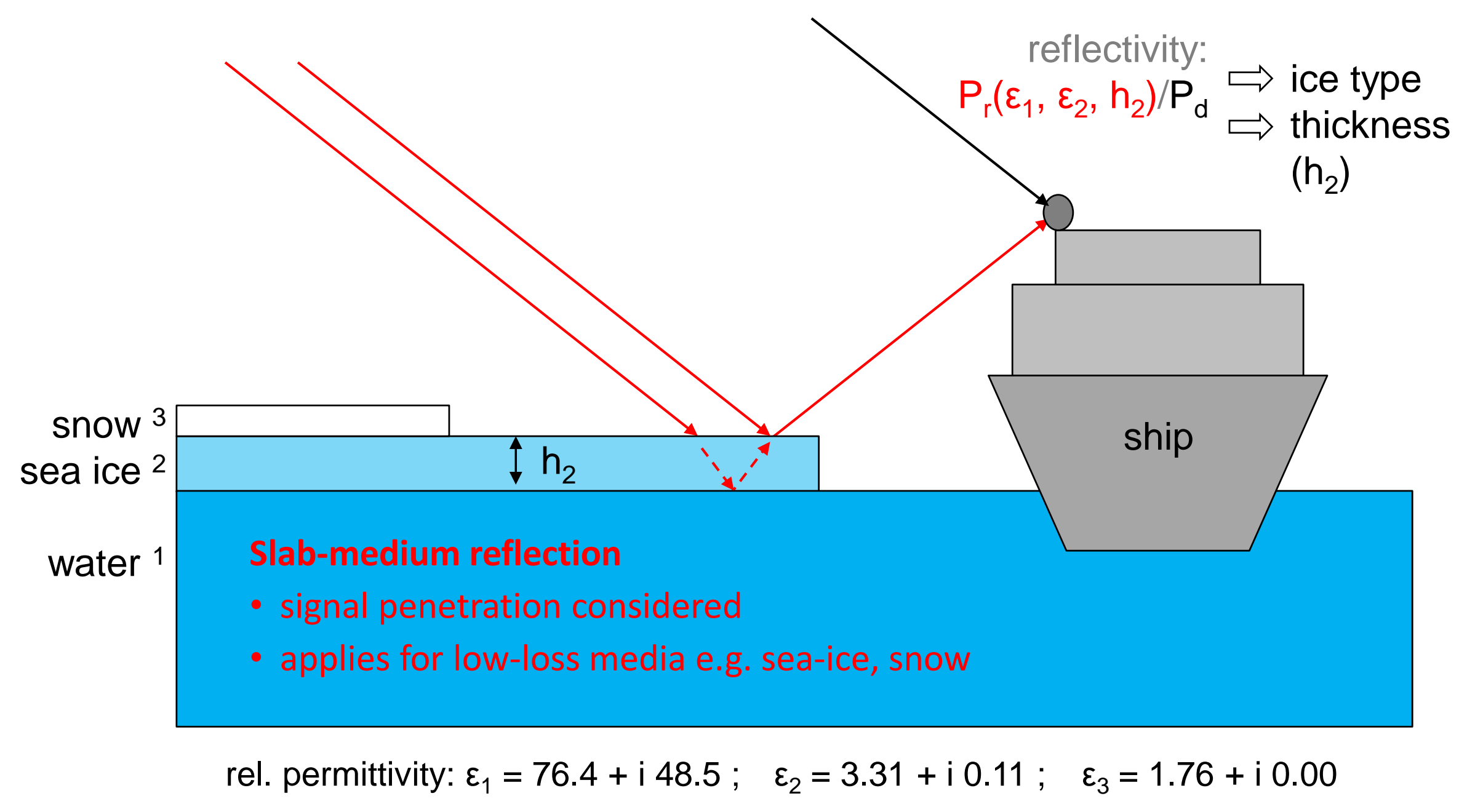




\section{Reflection Model}

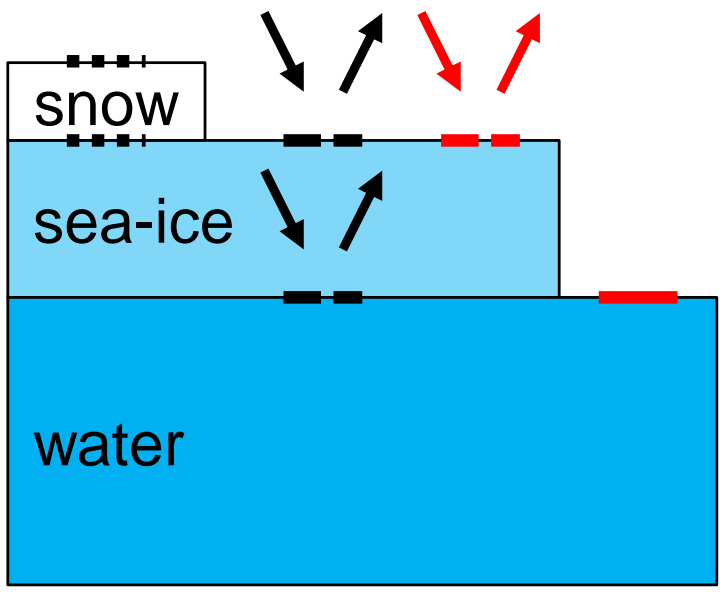

Bulk-medium reflection

Slab-medium reflection

Kaleschke et al. 2010

Water (W)

$\varepsilon=76.4+\mathrm{i} 48.5$

at $2^{\circ} \mathrm{C}$

"opaque"

"opaque“

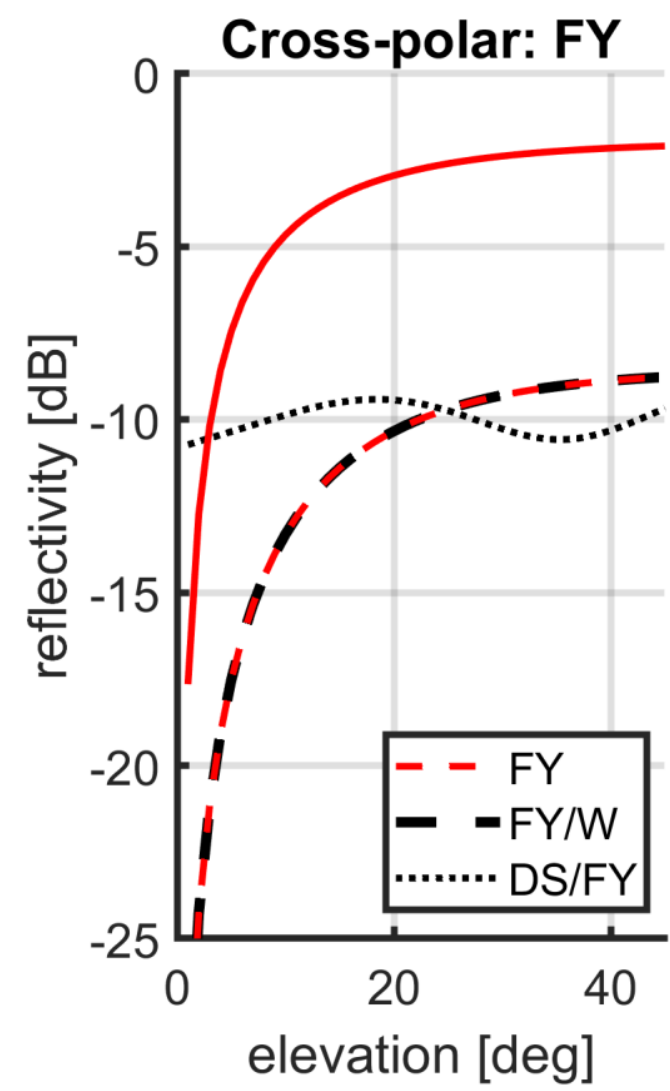

First-year (FY) ice type:

$\varepsilon=4.75+\mathrm{i} 0.91$

at $-1^{\circ} \mathrm{C}, 1 \mathrm{~m}$ thick
Multiyear (MY) ice type:

$\varepsilon=3.31+\mathrm{i} 0.11$

at $-1^{\circ} \mathrm{C}, 1 \mathrm{~m}$ thick

„transparent"
Semmling et al. (under review)

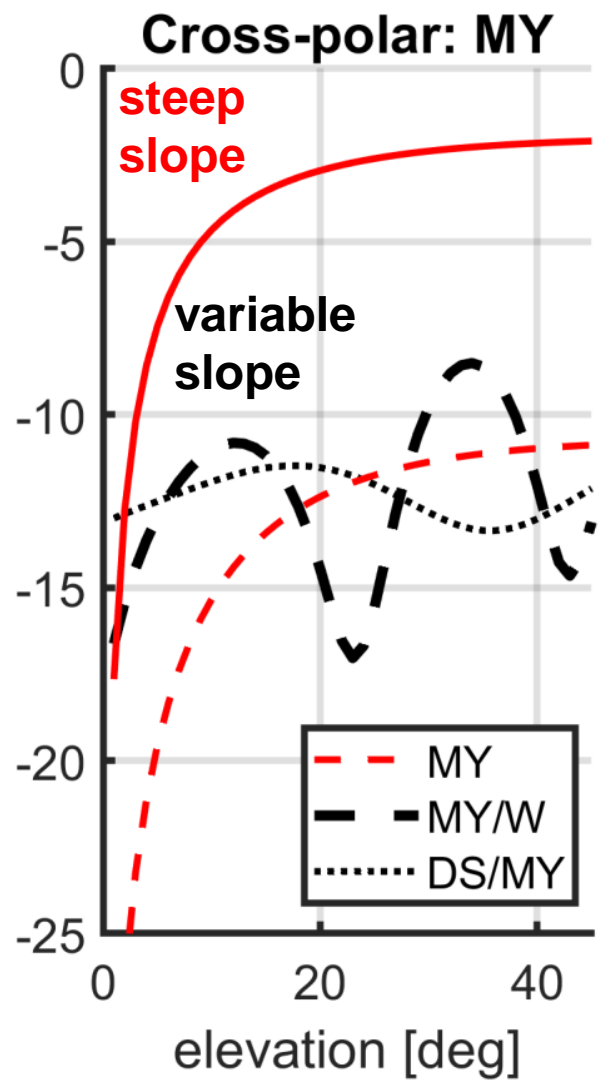

\section{GF Z}

DLR Helmholtz Centre
POTSDAM 


\section{Results for MOSAiC (first leg)}




\section{Reflectivity Profiles}

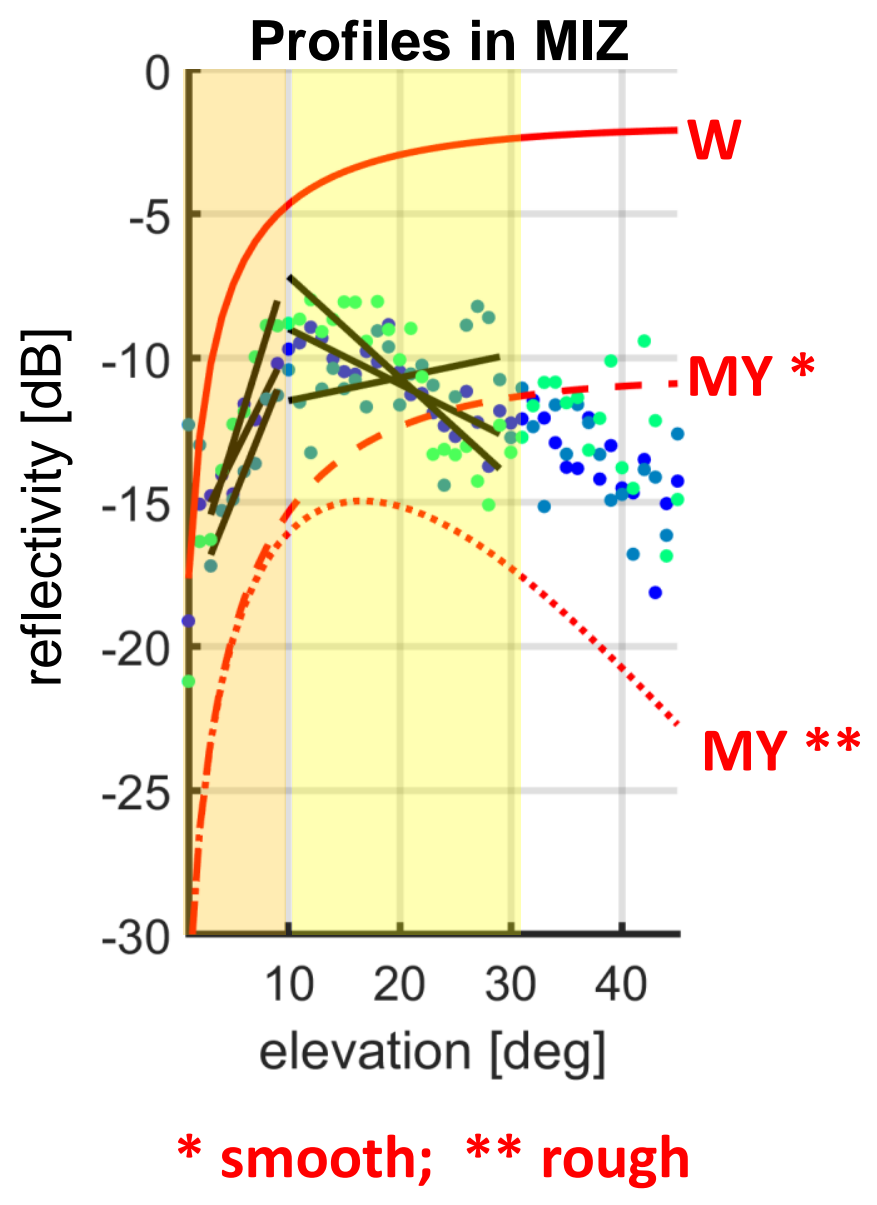

- obs. (day color-coded)
Low-Elevation Range $\left(1^{\circ}\right.$ to $\left.10^{\circ}\right)$

- reflect. between MY and W

- steep slope of bulk model

- no roughness effect

Mid-Elevation Range $\left(10^{\circ}\right.$ to $\left.30^{\circ}\right)$

- reflect. above MY

- moderate slope (decrease)

- small roughness effect

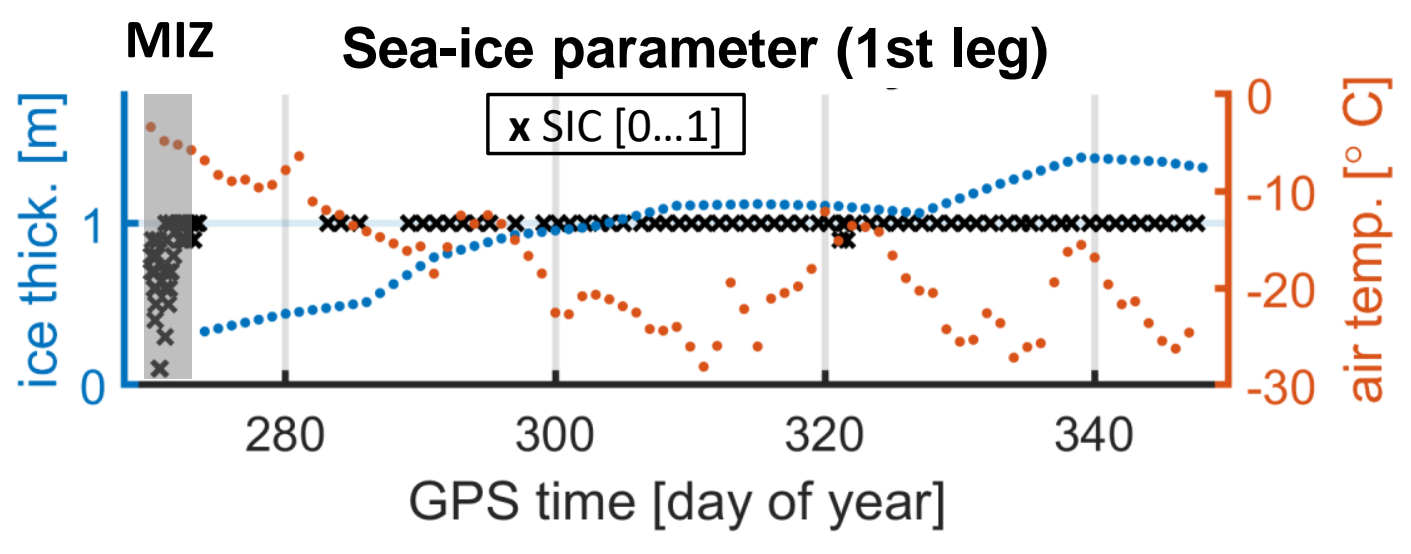




\section{Reflectivity Profiles}

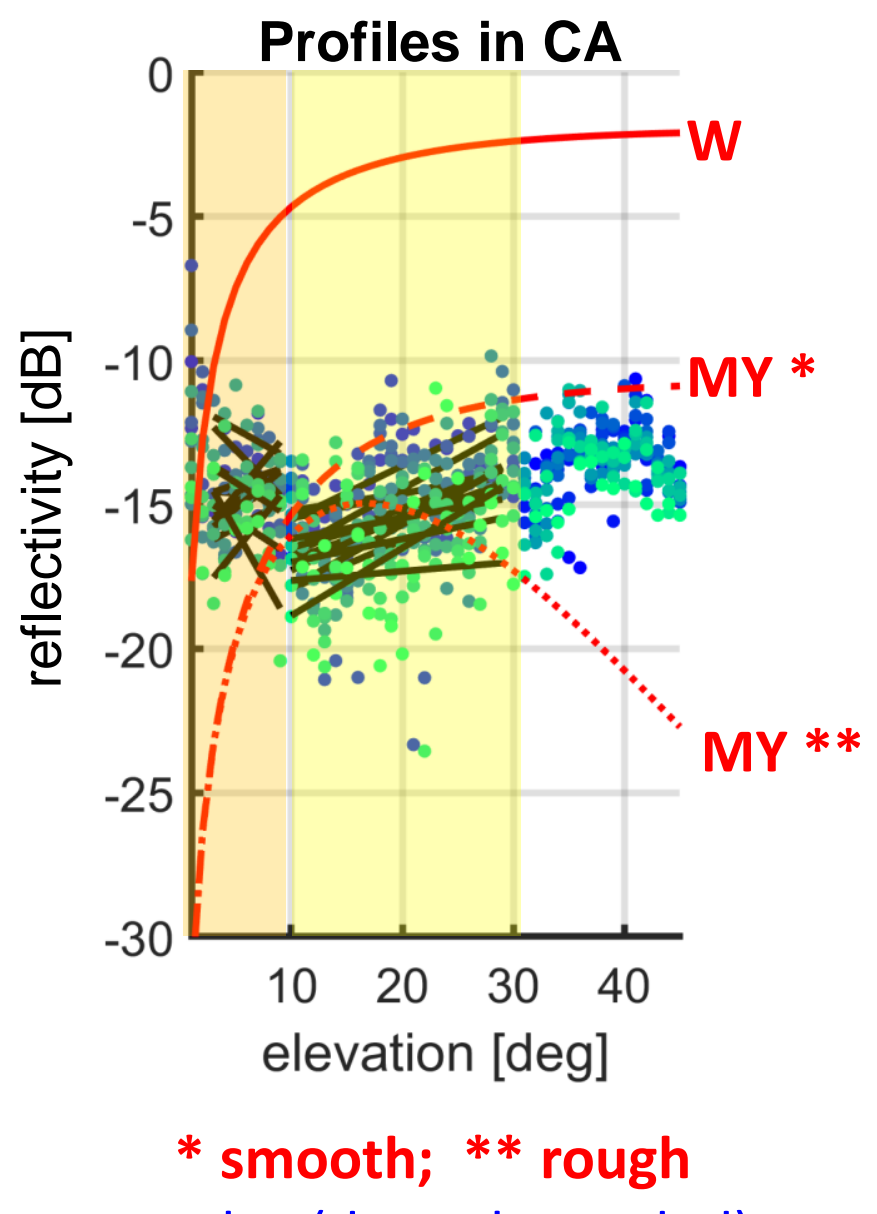

- obs. (day color-coded)
Low-Elevation Range $\left(1^{\circ}\right.$ to $\left.10^{\circ}\right)$

- reflect. between MY and W

- slope deviates from bulk model

- no roughness effect

Mid-Elevation Range $\left(10^{\circ}\right.$ to $\left.30^{\circ}\right)$

- reflect. below MY

- slope of slight increase

- no roughness effect permittivity inversion

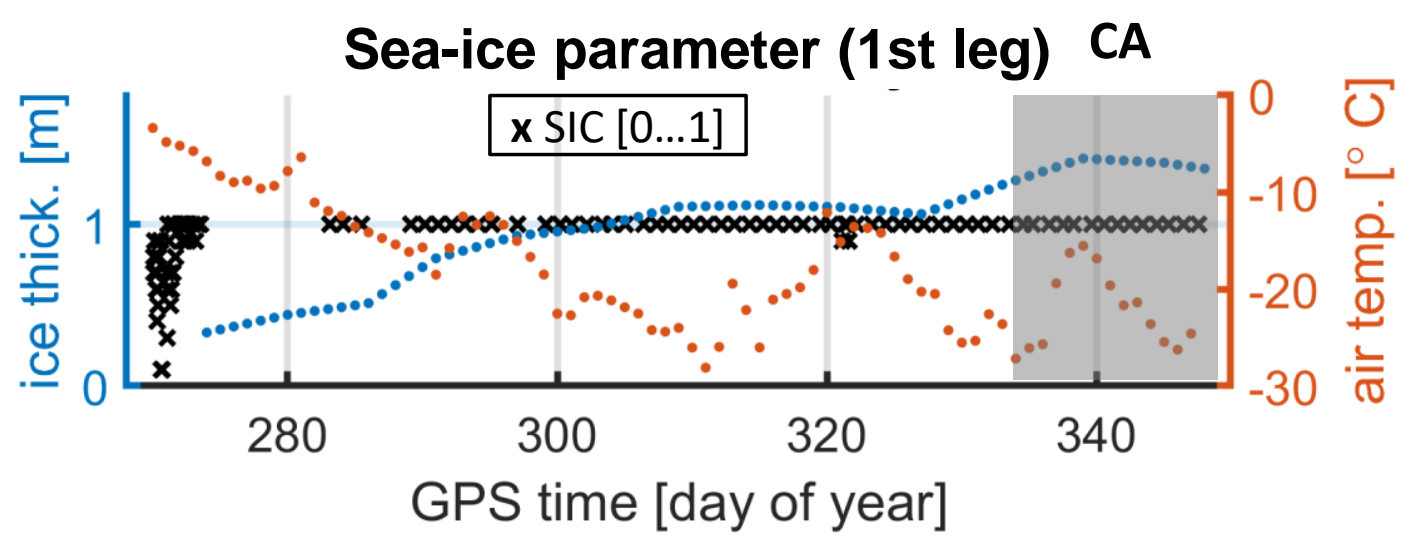

\section{G F Z}




\section{Inverted Permittivity}
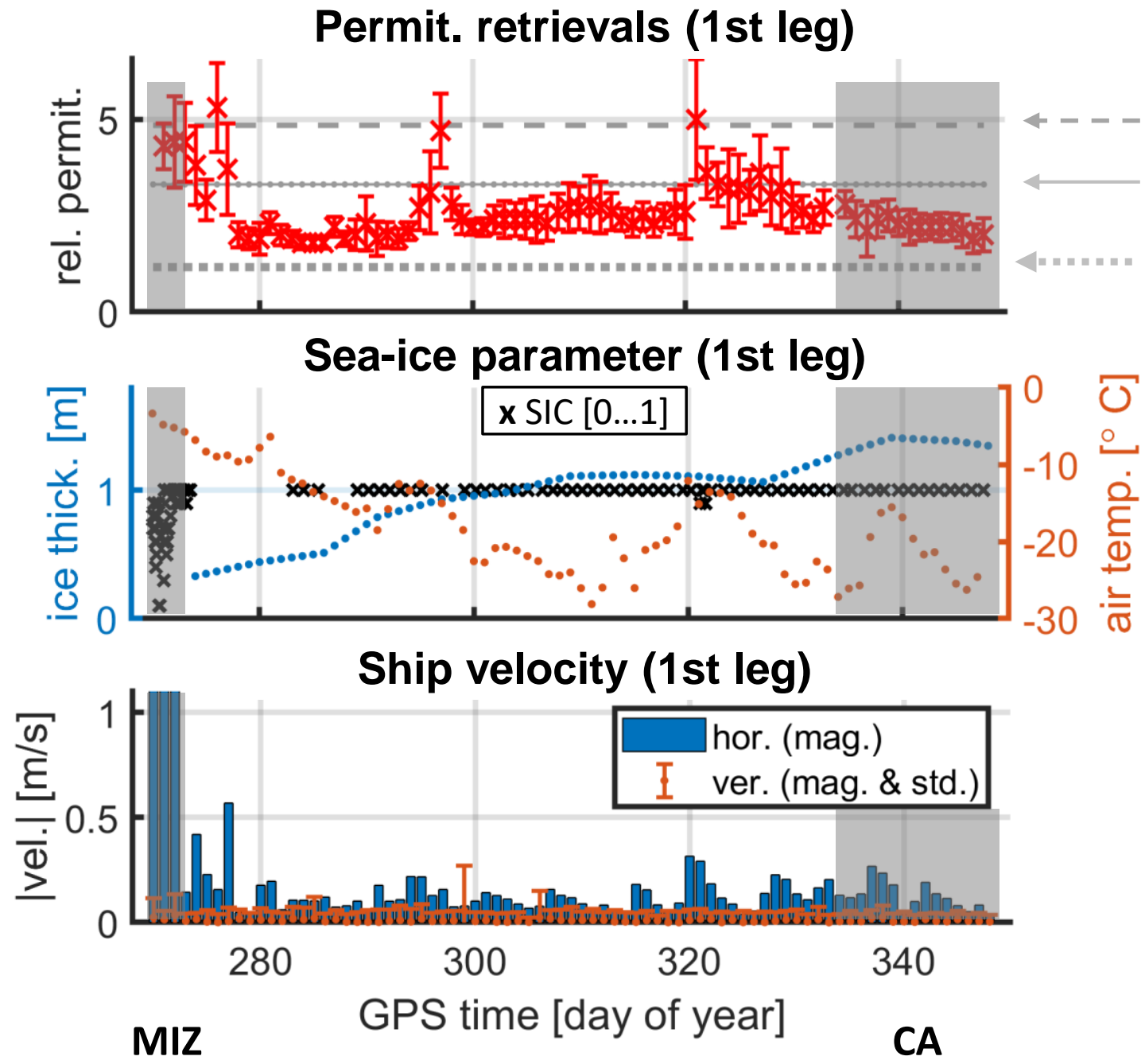

FY ice

MY ice

Dry snow

In Marginal Ice Zone

- $\varepsilon$-estimates rather high ( $F Y$ to $M Y$ range)

- water occurrence $(\mathrm{SIC}<1)$

\section{In Central Arctic}

- $\varepsilon$-estimates low (MY to DS level)

- compact ice (SIC 1)

- thickness $>1 \mathrm{~m}$

\section{In general}

- estimates often close to DS level impact of snow cover?

- water occurrence $(\mathrm{SIC}<1)$ has major impact (estimate increase)

- thickness has not major impact

\section{G F Z}




\section{Slope Anomalies to be analysed ...}
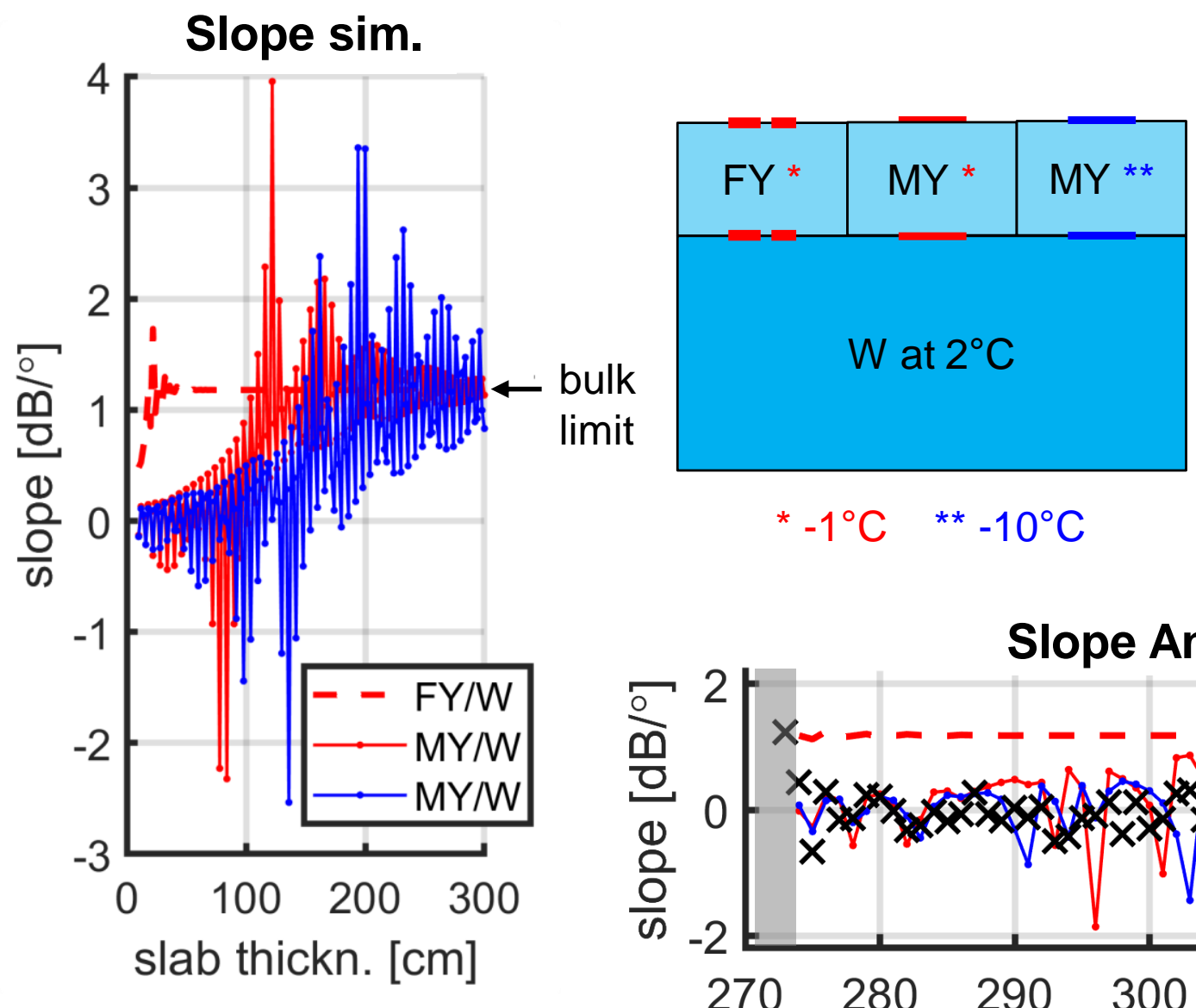

FY ice slab

- small anomaly range

- bulk limit reached $\mathrm{h}>20 \mathrm{~cm}$

MY ice slab $\left(-1^{\circ} \mathrm{C}\right)$

- large anomaly range

- bulk limit reached $\mathrm{h}>110 \mathrm{~cm}$

MY ice slab $\left(-10^{\circ} \mathrm{C}\right)$

- even larger anomaly range

- bulk limit reached $h>140 \mathrm{~cm}$

- best agreement with slope estim.

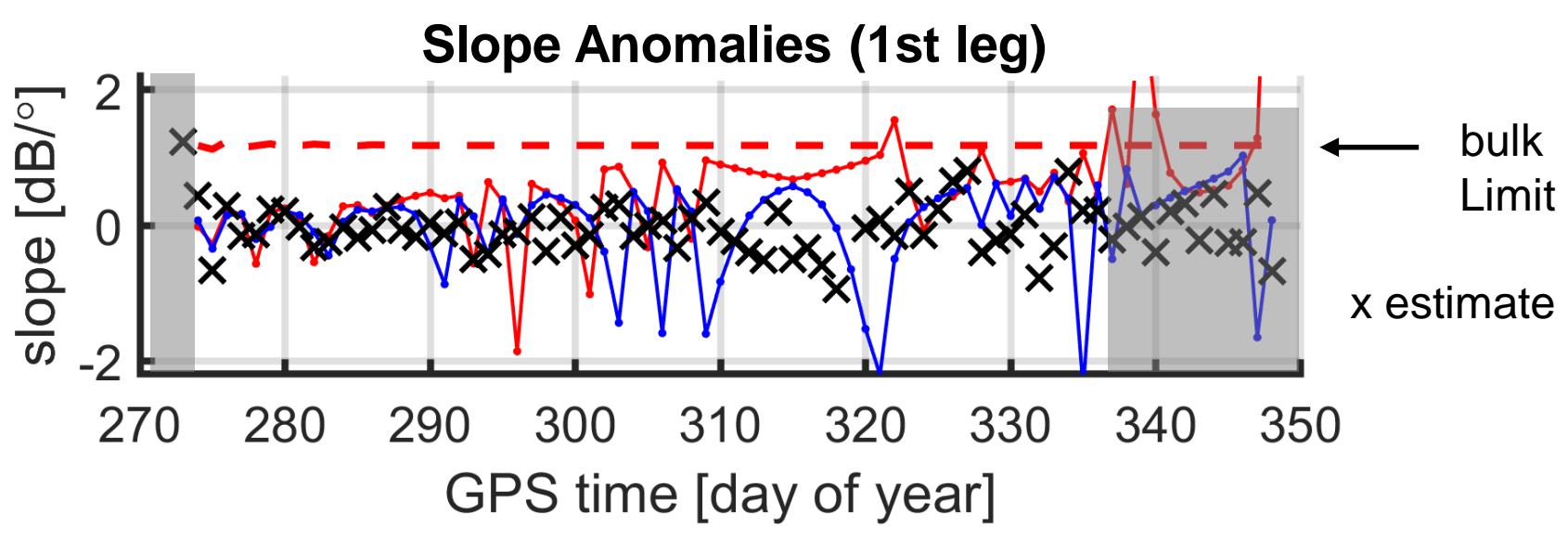

\section{GF Z}

DLR Helmholtz Centre 


\section{Summary \& Conclusions}

More MOSAiC: Dec 2019 - Jun 2020

\section{Permittivity Estimation}

- Can we link estimates and ice type?

- Estimates range exceeds ice type values

- impact of sea-ice concentration dominates

\section{Slope Anomalies}

- Charact. bulk profiles in MIZ

- Significant slope anomaly in CA

- Ice thickness inversion difficult

- Anomalies sensitive to ice types

\section{More data available}

- for MOSAiC's second leg

- from central Arctic to Fram Strait

- 171 days (Dec 15, 2019 - Jun 2, 2020)

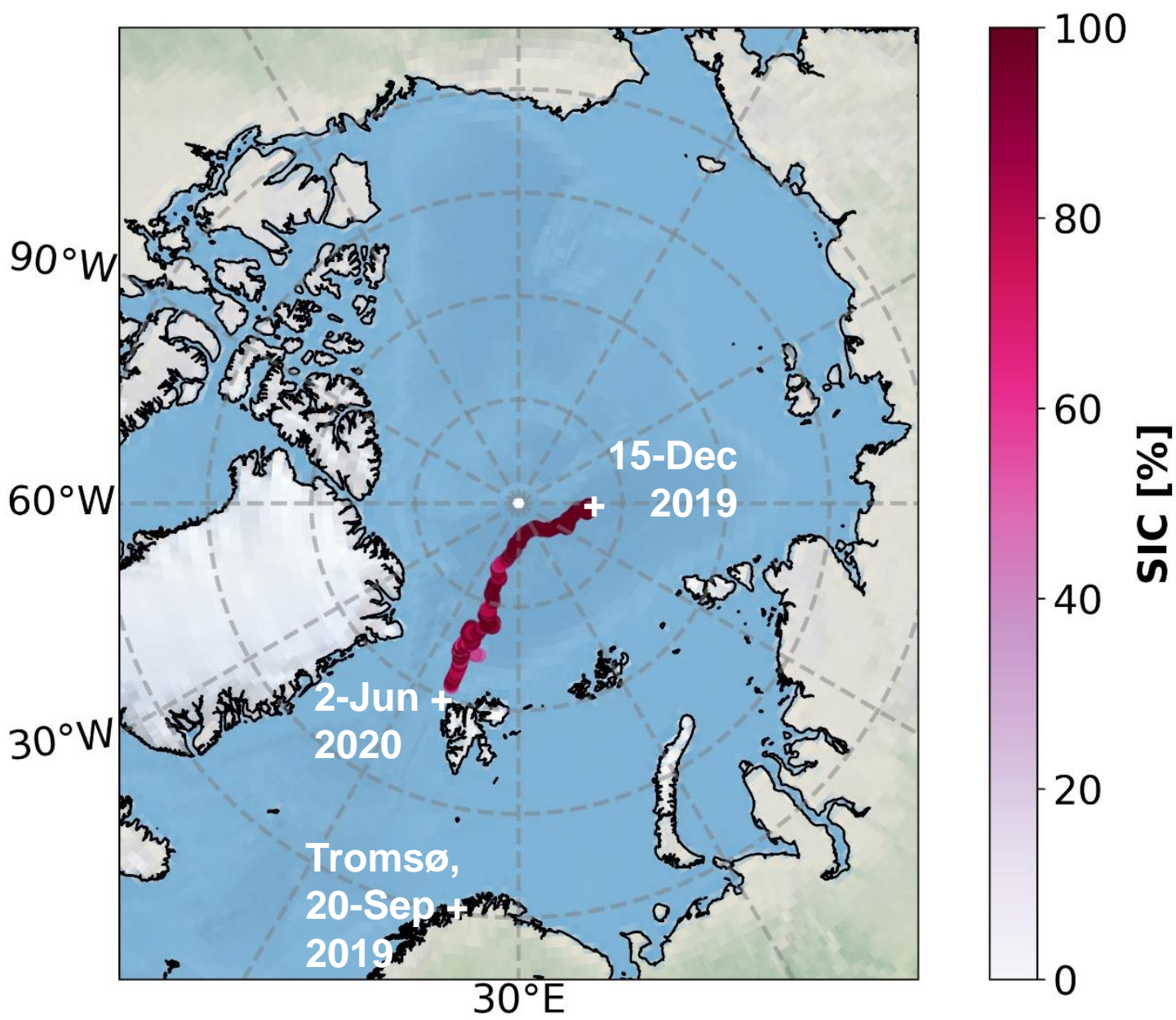

\section{G F Z}




\section{References}

- Helm et al. 2007: GORS - A GNSS Occultation, Reflectometry and Scatterometry Space Receiver. ION GNSS 2007

- Kaleschke et al. 2010: A sea-ice thickness retrieval model for $1.4 \mathrm{GHz}$ radiometry and application to airborne measurements over low salinity sea-ice.

The Cryosphere

- ASSIST 2016: Arctic shipborne sea ice standardization tool. Technical report, International Arctic Research Center, http://www.iarc.uaf.edu/icewatch

- Cardellach et al. 2018: GNSS Transpolar Earth Reflectometry exploriNg System (G-TERN): Mission Concept. IEEE Access

- Semmling et al. 2019: Sea Ice concentration derived from GNSS reflection measurements in Fram Strait. IEEE Trans. Geosci. Rem. Sens.

- Munoz-Martin et al. 2020: Snow and Ice Thickness Retrievals Using GNSS-R: Preliminary Results of the MOSAiC Experiment.

Remote Sensing

- AWI 2020: Weekly combined SMOS/CryoSat-2 product, available via https://data.meereisportal.de

- ECMWF 2020: ERA-5 reanalysis data, available via https://cds.climate.copernicus.eu

- Semmling et al. (under review): Sea-ice permittivity derived from GNSS reflection profiles: Results of the MOSAiC expedition.

IEEE Trans. Geosci. Rem. Sens. 
